\title{
Patient preferences on telemedicine for preanesthesia evaluation
}

\author{
Michael Fishman, MD · Brian Mirante, MD • \\ Feng Dai, PhD $\cdot$ Viji Kurup, MD
}

Received: 11 September 2014/ Accepted: 19 November 2014/Published online: 26 November 2014

(C) Canadian Anesthesiologists' Society 2014

\section{To the Editor,}

Telemedicine is gaining traction in both medical and surgical specialties. Three recent pilot studies reported success in using dedicated telemedicine setups, including an electronic stethoscope and airway camera, for preanesthesia consultation by anesthesiologists. ${ }^{1-3}$ The most recent study randomized 155 head and neck surgery patients to undergo either an in-person or a telemedicine preanesthesia evaluation. ${ }^{3}$ The investigators reported equivalent patient and provider satisfaction as well as concordance with the physical exam when compared with the day of surgery. ${ }^{3}$

We conducted a study investigating patient preferences regarding the use of telemedicine for the preoperative anesthesia assessment as well as patient opportunity costs to attend the preanesthesia clinic. The motivation for our study was based on the authors' observation that many preanesthesia clinic (PAC) patients were American Society

Parts of this data set were presented in abstract format at the American Society of Anesthesiologists' annual meeting, October 2014.

\section{Fishman, MD ( $\square)$.}

Department of Anesthesiology, Perioperative and Pain Medicine, Stanford University, Redwood City, CA, USA

e-mail: MAFishman@gmail.com

\section{B. Mirante, MD}

Middlesex Hospital, Middletown, CT, USA

F. Dai, PhD

Yale Center for Analytical Sciences, Yale University,

New Haven, CT, USA

V. Kurup, MD

Department of Anesthesiology, Yale University, New Haven, CT, USA of Anesthesiologists I or II physical status and oftentimes reported financial and time loss to attend the PAC. The location for this study was a major academic tertiary referral centre performing nearly 40,000 inpatient and outpatient procedures annually.

Following exemption from the Institutional Review Board review by the Yale University Human Investigation Committee in April 2012, patients at the PAC were invited to complete a nine-item questionnaire prior to their appointment. An explanation of the project and a photo depiction of the telemedicine encounter were provided. Data collected included: distance travelled (miles), travel time (minutes), out-of-pocket expenses (USD), separate physician visits for upcoming surgery, willingness to videoconference $(\mathrm{Y} / \mathrm{N})$, and preference for visiting an alternate facility. The continuous data were evaluated using Wilcoxon rank-sum test, and the categorical variables were evaluated using the Fisher's exact test. A Bonferroni correction method was used to adjust for multiple comparisons. All reported $P$ values are two sided. All statistical analyses were performed using the statistical software SAS ${ }^{\circledR}$, version 9.3 (Cary, NC, USA).

Seven hundred seventy-seven surveys were administered with a response rate of $94.5 \%$. The average age of respondents was $54.9 \mathrm{yr}$. Of the 728 respondents that documented a preference, $393(53.9 \%)$ were willing to videoconference. There was no association between measured variables and willingness to teleconference at the $a$ priori level of significance.

The results from our study show a divided willingness on the part of our patient population to undergo the preanesthetic evaluation using telemedicine. Commentary on the survey from patients unwilling to videoconference suggested that many continued to have misconceptions regarding the proposed telemedicine encounter despite use 
Figure Willingness to videoconference based on patient opportunity cost in terms of time, travel, and out-ofpocket expenses. Those who were willing to videoconference had marginally longer mean (SD) travel minutes than those who were unwilling $(P=0.029)$, but this did not reach the $a$ priori multiplicity-corrected threshold for statistical significance
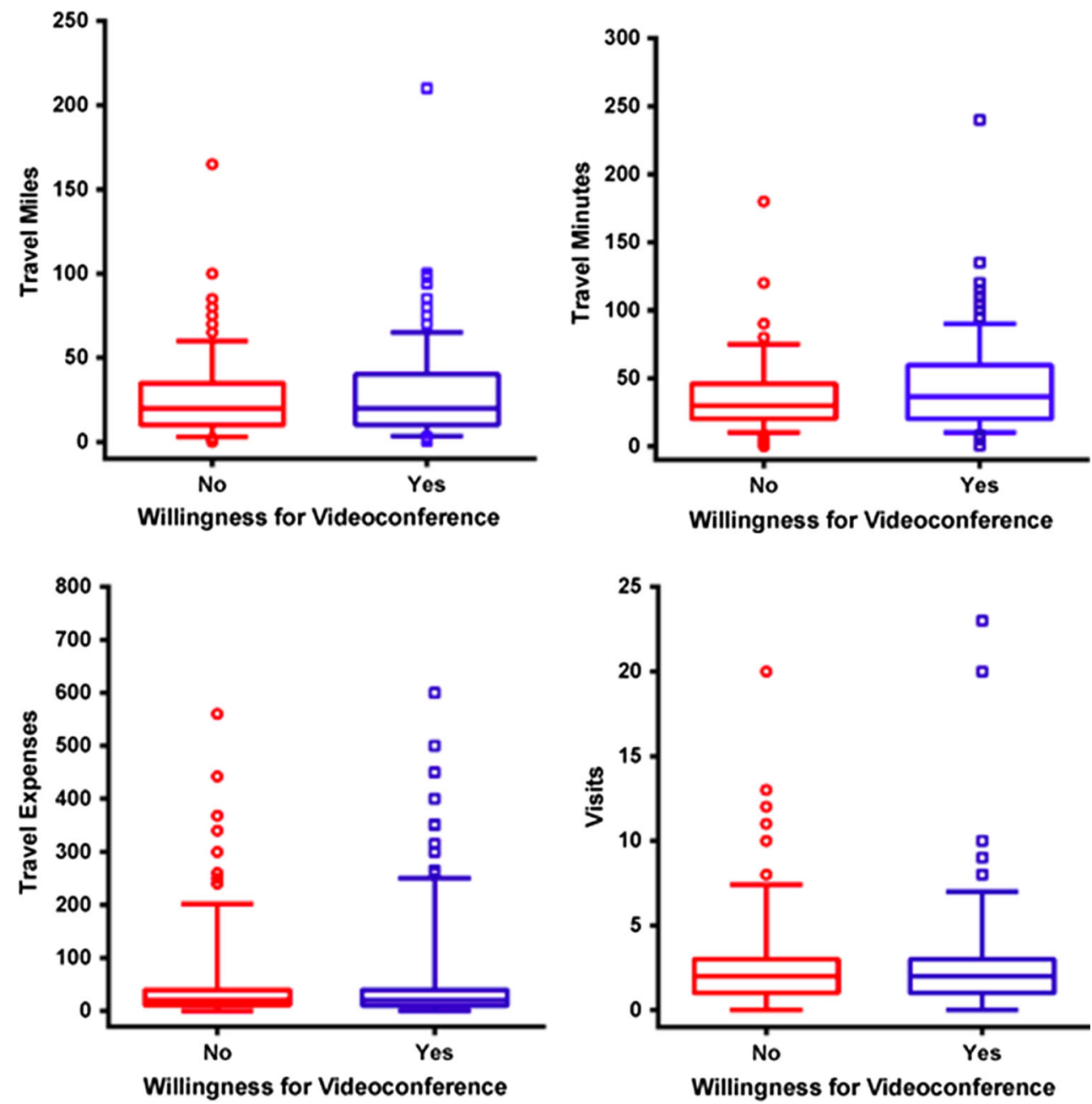

of a scripted explanation and depiction. Such commentary included "I prefer to talk to a person", "I have already travelled here", "Privacy concerns", and "I don't want to be videotaped".

It is interesting and counterintuitive that there was no statistically significant association between willingness to videoconference and patient opportunity costs. We expected that patients who faced long travel times and distances as well as greater costs to be more willing to videoconference. We speculate that the timing of the survey prior to the encounter made it difficult for patients to answer, as they were asked to comment on something they had not yet experienced. Additionally, we did not assess patients' prior experience with videoconferencing or use of other electronic services, such as banking. In this day and age where nearly every service can be conducted online, perhaps medicine is the final frontier where the intangible, but real, power of the human touch is still meaningful.
Acknowledgements Audrey Senior, APRN; Craig Odermatt, BS; Josephine Pinto; Janet Punzelt, RN for their contributions in coordinating data collection.

Conflicts of interest None declared.

\section{References}

1. Wong DT, Kamming D, Salenieks ME, Go K, Kohm C, Chung F. Preadmission anesthesia consultation using telemedicine technology: a pilot study. Anesthesiology 2004; 100: 1605-7.

2. Zetterman CV, Sweitzer BJ, Webb B, Barak-Bernhagen MA, Boedeker $B H$. Validation of a virtual preoperative evaluation clinic: a pilot study. Studies Health Technol Inform 2011; 163 : 737-9.

3. Applegate RL 2nd, Gildea B, Patchin R, et al. Telemedicine preanesthesia evaluation: a randomized pilot trial. Telemed J E Health 2013; 19: 211-6. 